\title{
COMETARY VOLATILES
}

The status after comet C/1996 B2 Hyakutake

\author{
D. BOCKELEE-MORVAN \\ Observatoire de Paris \\ F-92195 Meudon Cedex, France
}

\begin{abstract}
Comets are believed to be the most pristine material surviving from the origin of the Solar System. The nature of their volatiles should in principle provide chemical and physical clues to their formation environment in the solar nebula. Another important aspect of the study of comets is the extent to which they may incorporate relatively unprocessed interstellar material.

Our knowledge of the composition of cometary ices has been greatly improved in this last decade. The years 1995-1996 were rich in new results, with the close approach to Earth of comet C/1996 B2 Hyakutake and the discovery of the bright comet $\mathrm{C} / 1995 \mathrm{O} 1$ Hale-Bopp at large heliocentric distance. We will present the current knowledge of the nature and abundances of cometary volatiles and mention some of the outstanding issues related to the origin of comets and their links to interstellar material.
\end{abstract}

\section{Introduction}

Comets are most likely important links in the chain that leads from protoplanetary disks to planets. Indeed, they are believed to be the remnants of the planetesimals whose agglomeration lead to the formation of planets. The standard picture is the origin of cometary nuclei from accretion of grains in the solar nebula.

The presence of volatile ices in cometary nuclei attests that they formed in the outer regions of the solar nebula. It is believed that new comets coming from the Oort cloud formed in the Uranus-Neptune zone. They were dynamically scattered to distant orbits by the growing proto-planets to form the Oort cloud. The perturbations induced at close encounters with Jupiter and Saturn tend to eject a majority of the planetesimals formed in their accretion zones into hyperbolic orbits. 
Short-period comets of the so-called Jupiter family presumably have formed beyond Neptune, in the Kuiper Belt whose first member was discovered in 1992 (Jewitt \& Luu 1993; Jewitt et al. 1996). The existence of this cometary reservoir was first postulated by Edgeworth (1949) and Kuiper (1951), as no reason was found for accretion of comet-size planetesimals to stop beyond Neptune. It was proposed as the best alternative source for the short period comets with prograde and low-inclination orbits, which the Oort cloud cannot adequately supply (Fernandez 1980; Duncan et al. 1988). Kuiper Belt objects are believed to possess interiors rich in volatile ices, commensurate with their accumulation in the cold outer regions of the solar nebula.

Due to their small size and long term residence in the outer regions of the Solar System, cometary nuclei did not undergo significant thermal or chemical processing since their formation. Cosmic ray irradiation only affected the composition of the surface, forming a carbon-rich refractory crust which is blown off as comets penetrate the inner solar system. It is believed that their study should tell us about the condensation and accretion processes which governed their formation, $4.5 \mathrm{Gyr}$ ago. Constraints on the physical conditions of the low-temperature regions in the solar nebula may be anticipated, as well as clues to the evolution of matter at the stages from interstellar clouds to planetary system.

In particular, comets may have preserved presolar matter from the parent interstellar cloud. During the collapse of the interstellar cloud, there might have been a region in the solar nebula where the interstellar grains coated with ice mantles survived. From comparison of the abundances of cometary and interstellar molecules, and the strong analogy between interstellar and cometary grains, Greenberg (1982) proposed that comets are made of unprocessed interstellar material. Yamamoto et al. (1983) and Yamamoto (1985), however, suggested that the formation region of comets underwent sufficient heating to sublimate the very volatile species $(\mathrm{CO}$ and $\mathrm{N}_{2}$ ) of the ice mantles. Lunine et al. (1991) proposed that the heating could have been more substantial. Cometary ices could be ices of second generation which recondensed onto the cold grains. At the opposite, for Lewis \& Prinn (1980), cometary ices are condensates of an initially hot solar nebula.

In this paper, we review our present knowledge of the composition of cometary volatiles. Some of the outstanding issues in the framework of the origin of cometary material will be given. 


\section{Molecular identifications and abundances}

Several recent review papers already focussed on the composition of cometary volatiles. In particular, Crovisier (1994) made an exhaustive inventory of known cometary parent molecules, with a critical review of the evaluations of their abundances. Mumma et al. (1993a) discussed in addition the implications for the origin of comets and their possible link to the interstellar medium. This topic was also part of the comprehensive review of Festou et al. (1993). Recently, two bright comets made spectacular apparitions. C/1996 B2 Hyakutake approached at only 0.1 AU from the Earth in March 1996, and extensive spectroscopic studies resulted in the detection of new species. C/1995 O1 Hale-Bopp was detected and found to be exceptionnally active at $7 \mathrm{AU}$ from the Sun in July 1995: many species were observed at large heliocentric distances, bringing unprecedented insights on the sublimation processes of cometary ices. This paper will emphasize these new results.

\subsection{METHODS}

Cometary ices are generally not accessible directly. Cometary nuclei are kilometre-sized bodies which develop an atmosphere rich in gas and dust, as they approach the Sun. This bright coma prevents the observation of weak spectral signatures coming from the ices. The only reported detections are the broad band absorption features at 3.0 and $2.0 \mu \mathrm{m}$, attributed to water-ice grains in the coma of comet P/Halley (Combes et al. 1988) and C/1995 O1 Hale-Bopp (Davies et al. 1995), respectively.

Cometary ices can be probed from the composition of the gaseous coma. The most direct method is the observation of the so-called parent molecules which directly outgas from the nucleus, although their abundance in the coma may not directly reflect the nucleus volatile composition, due to sublimation fractionation effects. A decade ago, no cometary parent molecule was directly observed, except the $\mathrm{CO}$ and $\mathrm{S}_{2}$ molecules detected in the UV. Indirect identifications were provided by the observations of their photodissociation and photoionisation products (e.g. $\mathrm{H}_{2} \mathrm{O}$ from $\mathrm{OH}$ in the UV or radio, $\mathrm{CO}_{2}$ from $\mathrm{CO}_{2}^{+}$in the visible). The in situ exploration of comet $\mathrm{P} /$ Halley and the opening up of the infrared and microwave domains allowed the quest for parent molecules. Indeed, most parent molecules have weak spectral signatures in the UV or visible, but their vibrational bands and rotational lines at infrared and radio wavelengths are in general much stronger. Progress made in identifications coincides not only with the opportunity of bright comets but also with improvements of observational techniques such as the availability of new receivers in the microwave or echelle spectrographs in the infrared domains. 
TABLE 1. Molecular abundances in comets.

\begin{tabular}{|c|c|c|c|c|}
\hline Molecule & $\begin{array}{l}\mathrm{T} \\
{[\mathrm{K}]}\end{array}$ & $\begin{array}{l}\mathrm{C} / \text { Hyakutake } \\
\text { at } 1 \mathrm{AU}\end{array}$ & $\begin{array}{l}\text { Others } \\
\text { at } 1 \mathrm{AU}\end{array}$ & $\begin{array}{l}\text { C/Hale-Bopp } \\
\text { at } 4 \mathrm{AU}\end{array}$ \\
\hline $\mathrm{H}_{2} \mathrm{O}$ & 152 & 100. & 100. & 100. \\
\hline $\mathrm{CO}$ & 24 & 5.-30. & $2 .-20$. & 80. \\
\hline $\mathrm{CO}_{2}$ & 72 & $\leq 7$ & 3.-6. & $30 .^{a t 4.6 A U}$ \\
\hline $\mathrm{CH}_{4}$ & 31 & 0.7 & $\leq 0.5-2$ & \\
\hline $\mathrm{C}_{2} \mathrm{H}_{2}$ & 54 & $0.3-0.9$ & & \\
\hline $\mathrm{C}_{2} \mathrm{H}_{6}$ & 44 & 0.4 & & \\
\hline $\mathrm{CH}_{3} \mathrm{OH}$ & 99 & 2. & 1.-7. & 6. \\
\hline $\mathrm{H}_{2} \mathrm{CO}$ & 64 & $0.2-1$ & $0.05-4$ & $0.1-0.2$ \\
\hline $\mathrm{NH}_{3}$ & 78 & 0.5 & $0.4-0.9$ & \\
\hline $\mathrm{N}_{2}$ & 22 & & 0.02 & \\
\hline $\mathrm{HCN}$ & 95 & 0.15 & $0.1-0.2$ & 0.6 \\
\hline $\mathrm{HNC}$ & & 0.01 & & \\
\hline $\mathrm{CH}_{3} \mathrm{CN}$ & 93 & 0.01 & & \\
\hline $\mathrm{HC}_{3} \mathrm{~N}$ & 74 & & $\leq 0.02$ & \\
\hline $\mathrm{H}_{2} \mathrm{~S}$ & 57 & 0.6 & 0.3 & 6. \\
\hline OCS & 57 & 0.3 & $\leq 0.5$ & \\
\hline $\mathrm{S}_{2}$ & & 0.005 & $0.02-0.2$ & \\
\hline $\mathrm{SO}_{2}$ & 83 & & $\leq 0.001$ & \\
\hline
\end{tabular}

Equilibrium sublimation temperatures (T) are from Yamamoto (1985), completed by Crovisier (1996, private communication). Abundances for comets at 1 AU from the Sun are from the compilation of Crovisier (1994). Some of the abundances listed for Hyakutake slightly differ from preliminary published values, due to the adoption of improved water production rates. Abundances in comet Hale-Bopp are from Biver et al. (1996a), except for $\mathrm{CO}_{2}$ (Crovisier et al. 1996). For $\mathrm{H}_{2} \mathrm{CO}$, the lower and upper values refer to abundances under the assumption of direct release from the nucleus and production from an extended source, respectively.

\subsection{THE STATUS AFTER COMET C/1996 B2 HYAKUTAKE}

Table 1 summarizes our present knowledge of cometary composition. Measurements were made essentially in comets at about $1 \mathrm{AU}$ from the Sun, where the activity is driven by the sublimation of water ice. This last point is important to mention. As shown in Table 1 and discussed in Section 2.3, the coma of comet Hale-Bopp farther from the Sun exhibits quite a different composition.

\subsubsection{Water and deuterated water}

Water is the dominant cometary volatile. It was found to constitute $80 \%$ (by number) of the whole cometary gas in comet P/Halley (Krankowsky et 


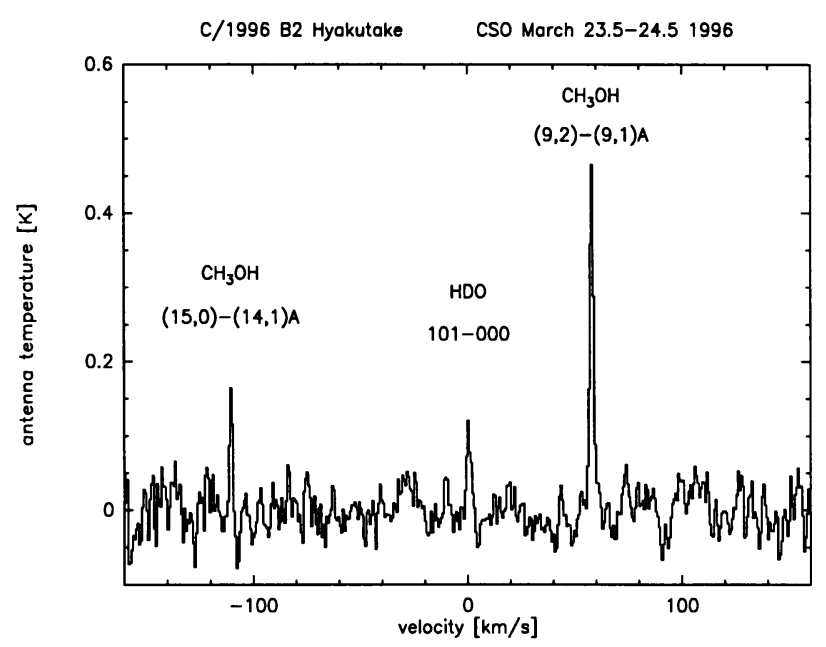

Figure 1. The HDO $1_{01}-0_{00}$ line at $465 \mathrm{GHz}$ and two methanol lines detected in comet C/1996 B2 Hyakutake on March 23-24, 1996 at the CSO (Lis et al. 1996a).

al. 1986). Water is well studied from the observation of its photodissociation product $\mathrm{OH}$ in the radio and in the UV. It was detected from space in two comets by mass spectroscopy (Krankowsky et al. 1986) and through the observation of its $\nu_{3}$ band at $2.7 \mu \mathrm{m}$ (Combes et al. 1988; Mumma et al. 1986). The detection of the $2_{02}-2_{03}$ ortho line of the $\nu_{1}+\nu_{2}-\nu_{1}$ vibrational band at $1.97 \mu \mathrm{m}$ in comet C/1992 XIX Shoemaker-Levy provided the first secure detection of cometary water from the ground (Mumma et al. 1995). Thirteen ro-vibrational lines of this band were detected in comet Hyakutake at the NASA IRTF telescope (Mumma et al. 1996a, b). This provided measurements of the rotational temperature and will presumably allow to constrain the abundance of the ortho and para spin species. From the relative intensities of the $\nu_{3}$ emission lines observed in comet $\mathrm{P} /$ Halley with the KAO, Mumma et al. (1987) deduced an ortho-to-para ratio of 2.5 which is equivalent to a spin temperature of $29 \mathrm{~K}$ and would suggest that cometary ices formed at low temperatures and preserved the spin temperature at which they were last processed. This study has been questioned however by Bockelée-Morvan \& Crovisier (1989) who retrieved from the same data a statistically equilibrated ortho-to-para ratio of 3 .

We might have some indirect evidences of the amorphicity of cometary water ice, a key clue to the origin of cometary material. Indeed, the crystallisation of amorphous water ice provides one of the best way to explain the sporadic and strong outbursts of comet P/Schwassmann-Wachmann 1 
along its distant and almost circular orbit (Espinasse et al. 1991; Cabot et al. 1996; Enzian et al. 1996), and the outburst of P/Halley at 14 AU from the Sun (Schmitt et al. 1993).

As will be discussed in Section 3, another fundamental constraint for the origin of comets is the $\mathrm{D} / \mathrm{H}$ ratio in water. Accurate determinations were obtained from the $\mathrm{H}_{2} \mathrm{DO}^{+} / \mathrm{H}_{3} \mathrm{O}^{+}$ratio measured by the mass spectrometer aboard Giotto (Eberhardt et al. 1995; Balsiger et al. 1995). Comet Hyakutake provided the first opportunity to search for cometary HDO from the ground. HDO was detected from its $1_{01}-0_{00}$ line at $465 \mathrm{GHz}$ at the CSO (Fig. 1; Lis et al. 1996a). Preliminary estimates provide a value in agreement with the value of $3.10^{-4}$ measured in P/Halley (Gautier et al. 1996). The detection of HDO in the IR has been announced by Mumma et al. (1996b) but the resulting $\mathrm{D} / \mathrm{H}$ ratio has not been yet published.

\subsubsection{Carbon monoxide}

$\mathrm{CO}$ is a major compound of cometary atmospheres. It was observed in the UV in several comets (Feldman 1991) and also studied by the NMS/Giotto spectrometer in $\mathrm{P} /$ Halley (Eberhardt et al. 1987). CO was first detected in the millimetre wavelength range in the distant comet $\mathrm{P} /$ SchwassmannWachmann 1 (Senay \& Jewitt 1994; Crovisier et al. 1995). Its rotational lines in the millimetre and submillimetre spectral window $(\mathrm{J}=1-0$ to $\mathrm{J}=5-4)$ were detected in comet Hyakutake at several telescopes and even mapped with the IRAM Plateau de Bure interferometer (Despois et al. 1996a). The fundamental vibrational band of $\mathrm{CO}$ at $4.7 \mu \mathrm{m}$ was securely detected for the first time in comet Hyakutake (Mumma et al. 1996a,b).

The reported $\mathrm{CO}$ abundances differ strongly. They range from $2 \%$ to 20-30\%. These abundance variations, which were measured in the coma, may not reflect only abundance differences in the nuclei but be partly due to the two-fold origin of CO. Indeed, Eberhardt et al. (1987) found that the spatial distribution of $\mathrm{CO}$ measured by the neutral mass spectrometer aboard Giotto was not consistent with direct release from the nucleus but required in addition a distributed source. The observations of $\mathrm{CO}$ in comet Hyakutake seem to support this two-fold origin. Indeed, from preliminary analyses, the IR (Mumma et al. 1996a), IUE (Feldman et al. 1996) and HST (Weaver et al. 1996a, b) observations yield abundances in the range 5-10\%, significantly below the abundances of 20-30\% derived from singledish radio observations (e.g. Matthews et al. 1996). Since these latter were made with larger fields of view, they could have been more sensitive to the distributed source. The interferometric observations of the $\mathrm{CO}(2-1)$ line at $230 \mathrm{GHz}$, whose analysis is still in progress, should help in the study of the relative contributions of the nuclear and distributed sources of CO (Despois et al. 1996a). We must note that the nature of the distributed source of $\mathrm{CO}$ 
is still unknown. In comet $\mathrm{P} / \mathrm{Halley}$, it was noted that $\mathrm{H}_{2} \mathrm{CO}$ photolysis could be a significant but not sufficient source (Meier et al. 1993). CO is possibly produced by complex species or released by the organic grains composed of CHON elements discovered in P/Halley.

\subsubsection{Carbon dioxide}

The abundance of $\mathrm{CO}_{2}$ has been measured in several comets, either from direct detection at $4.27 \mu \mathrm{m}$ from space (Combes et al. 1988; Crovisier et al. 1996), from mass spectroscopy (Krankowsky et al. 1986) or from observation of the forbidden CO Cameron band system around $200 \mathrm{~nm}$ (Weaver et al. 1994). This latter is partly due to $\mathrm{CO}$ prompt emission following the photodissociation of $\mathrm{CO}_{2}$. In comet Hyakutake, at least seven bands of this system were observed (Weaver et al. 1996b). The $\mathrm{CO}_{2}$ abundance ranges from 3 to $6 \%$ with respect to water in comets observed near the Sun (Table 1). ISO observations show that $\mathrm{CO}_{2}$, which is extremely volatile compared to $\mathrm{H}_{2} \mathrm{O}$, is one of the major species released by comet Hale-Bopp at large heliocentric distances (Crovisier et al. 1996). It is thus an important component of cometary ices.

\subsubsection{Methanol and formaldehyde}

Another major component of cometary atmospheres is $\mathrm{CH}_{3} \mathrm{OH}$. Methanol has been observed in several comets in the radio or infrared range (e.g. Fig. 1; Bockelée-Morvan et al. 1994 a,b; Hoban et al. 1991). Methanol was also detected from its protonated ion in Giotto mass spectra (Geiss et al. 1991; Eberhardt et al. 1991). The methanol abundance was found to vary from $1 \%$ to $7 \%$, depending upon the comet and even possibly with time.

Formaldehyde has an uncertain abundance, although its presence in cometary atmospheres is ubiquitous. Its spatial profile measured by the Giotto mass spectrometers in comet $\mathrm{P} /$ Halley requires a distributed source (Meier et al. 1993) and almost no direct release from the nucleus. Subsequently, $\mathrm{H}_{2} \mathrm{CO}$ was observed in the radio in several comets. The inferred abundances are typically $1 \%$, assuming production from an extended source. The abundances retrieved under the assumption of direct release from the nucleus $(\simeq 0.2 \%)$ confirm that $\mathrm{H}_{2} \mathrm{CO}$ is a minor component of cometary nuclei $(\leq 0.2 \%)$. $\mathrm{H}_{2} \mathrm{CO}$ may be released in the coma by the progressive sublimation of formaldehyde polymers (Huebner 1987; Meier et al. 1993).

\subsubsection{Hydrocarbons: $\mathrm{CH}_{4}, \mathrm{C}_{2} \mathrm{H}_{6}$ and $\mathrm{C}_{2} \mathrm{H}_{2}$}

The observational campaign of comet Hyakutake at the NASA IRTF telescope brought important insights in the nature and abundances of hydrocarbons. 

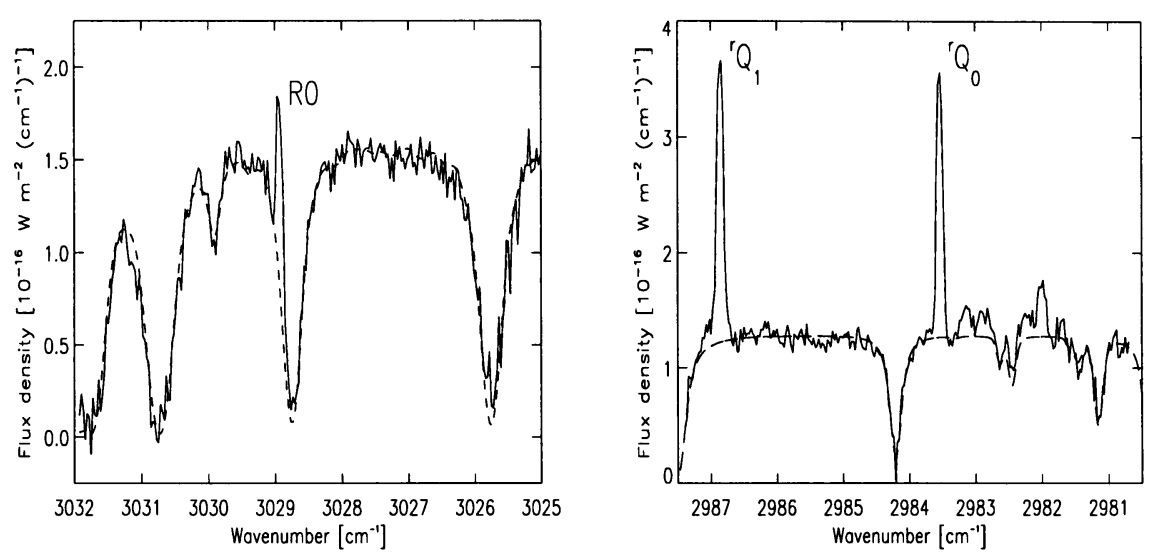

Figure 2. Infrared spectra of C/1996 B2 Hyakutake obtained at NASA IRTF on March 24,1996 . The telluric lines are seen in absorption against the cometary continuum (Mumma et al. 1996a); Left : The R0 line of the $\mathrm{CH}_{4} \nu_{3}$ band; Right: The ${ }^{r} \mathrm{Q}_{0}$ and ${ }^{r} \mathrm{Q}_{1}$ branches of the $\mathrm{C}_{2} \mathrm{H}_{6} \nu_{7}$ band.

Methane was definitely detected through several individual lines of its $\nu_{3}$ band at $3.3 \mu \mathrm{m}$ (Fig. 2). Early attempts in this infrared range lead only to upper limits or marginal detections. $\mathrm{CH}_{4}$ abundances of $0.5 \%$ (Haider et al. 1993 ) and $2 \%$ (Allen et al. 1987) were derived in comet $\mathrm{P} /$ Halley from ion mass spectrometer data, using different chemical models. The abundance of methane is estimated to $0.7 \%$ in comet Hyakutake (Mumma et al. 1996a).

Ethane was identified in comet Hyakutake through four Q branches of its $\nu_{7}$ band at $3.35 \mu \mathrm{m}$ (Mumma et al. 1996a, b; Fig. 2). Its abundance has been evaluated at $0.4 \%$ with respect to water. With such an abundance, ethane could contribute significantly to the 3.3-3.6 $\mu \mathrm{m}$ band observed in several comets with moderate spectral resolution.

The detection of $\mathrm{C}_{2} \mathrm{H}_{2}$ in comet Hyakutake is also a new result. Several emission lines in the $\nu_{3}$ fundamental at $3.0 \mu \mathrm{m}$ were identified (Fig. 3; Brooke et al. 1996). Its abundance is estimated between 0.3 and $0.9 \%$ with respect to water.

\subsubsection{The $3.2-3.6 \mu \mathrm{m}$ band: aliphatics and aromatics}

The origin of the $3.2-3.6 \mu \mathrm{m}$ emission band observed with moderate spectral resolution in a dozen of comets is still not completely explained. This band, which is characteristic of $\mathrm{C}-\mathrm{H}$ stretching modes, shows the signatures of both aliphatic and aromatic unsaturated carbonaceous compounds. Methanol has strong vibrational bands in this spectral region and contributes to about half of the observed emission. Residual emissions remain at $3.28,3.35$ and $3.43 \mu \mathrm{m}$. The $3.28 \mu \mathrm{m}$ feature, due to aromatics, is only 


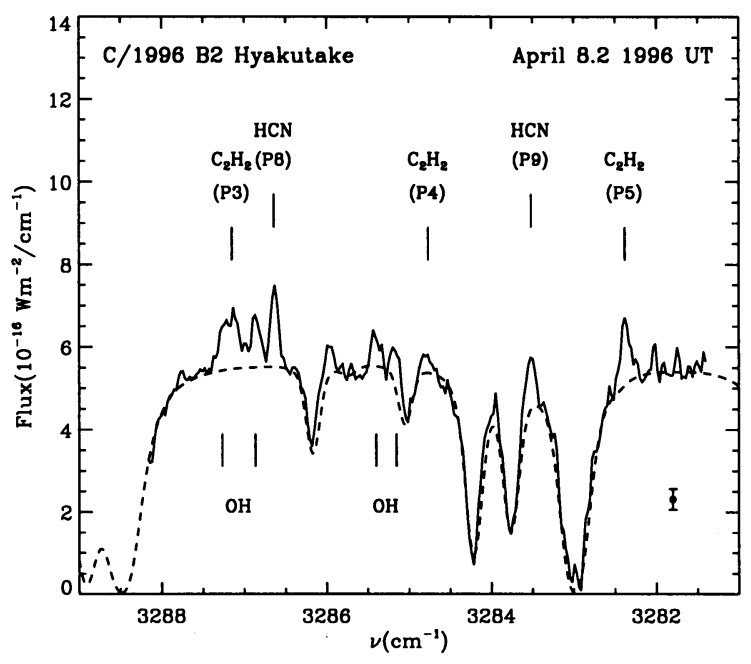

Figure 3. Detection of $\nu_{3}$ ro-vibrational lines of $\mathrm{C}_{2} \mathrm{H}_{2}$ and $\mathrm{HCN}$ in comet C/1996 B2 Hyakutake on April 8, 1996 at the NASA IRTF telescope (Brooke et al. 1996).

present in comets with high dust-to-gas ratios, suggesting a connection with the dust. Bockelée-Morvan et al. (1995) showed that it may be explained by a small abundance of PAHs of about $0.001 \%$, if it is emitted after electronic excitation in the UV followed by internal conversion to vibrational excitation. A PAH, phenanthrene, was indeed identified at $350 \mathrm{~nm}$ in the visible spectrum of $\mathrm{P} /$ Halley recorded by the TKS instrument on the VEGA probe (Moreels et al. 1994). The phenanthrene abundance derived from these UV observations is however estimated at $0.15 \%$. Joblin et al. (1996) found recently that the photodissociation lifetime of cometary PAHs is very short. If the emissions at $3.28 \mu \mathrm{m}$ and $350 \mathrm{~nm}$ are due to gas-phase PAHs, then a continuous production should exist. A realistic process is the progressive sublimation of dust grains, hence the connection with the dust. Detailed modelling is needed to investigate whether the IR and visible aromatics abundances can be reconciled with this process.

As discussed above, the $3.35 \mu \mathrm{m}$ residual emission feature is presumably the signature of ethane. The origin of the 3.4-3.5 broad band, which peaks at $3.43 \mu \mathrm{m}$, is however still unknown. From the analysis of the intensity of the band in a sample of comets at different heliocentric distances and water production rates, we have strong indications that the band arises mainly from gas-phase fluorescence (Bockelée-Morvan et al. 1995). It could be explained by a few percent of aliphatic species. DiSanti et al. (1995) observed part of this band at high spectral resolution in comet P/Swift- 


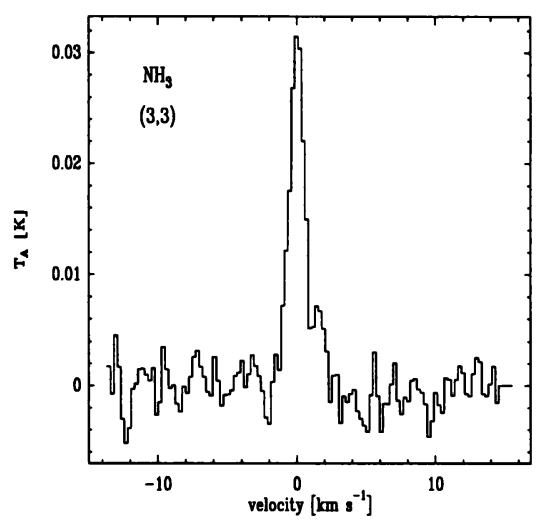

Figure 4. The $\mathrm{NH}_{3}(3,3)$ line at $23.9 \mathrm{GHz}$ detected in comet C/1996 B2 Hyakutake on March 24, 1996 with the NRAO 43-m telescope (Wootten et al. 1996a, b). The (1,1) line at $23.7 \mathrm{GHz}$ was also detected.

Tuttle and detected a narrow feature at $3.424 \mu \mathrm{m}$ suggestive of a Q-branch of a still unidentified molecular band. Progress on the origin of the $3.43 \mu \mathrm{m}$ band could come from further observations with high spectral resolution in bright comets. Laboratory spectra of C-H bearing species will be extremely desirable for line assignments.

\subsubsection{Nitrogen species}

Four nitrogen-bearing parent molecules, coming presumably from the nucleus, are now firmly identified from their spectral signatures: $\mathrm{NH}_{3}, \mathrm{HCN}$, $\mathrm{HNC}$ and $\mathrm{CH}_{3} \mathrm{CN}$.

First claimed to be detected in comet Iras-Araki-Alcock 1983 VII (Altenhoff et al. 1984), ammonia was definitely identified at centimetre wavelengths in comet Hyakutake with an abundance of about $0.5 \%$ relative to water (Fig. 4; Wootten et al. 1996a, b). The abundance of ammonia was estimated in several comets from the observation of its photodissociation products $\mathrm{NH}$ and $\mathrm{NH}_{2}$. From the analysis of $\mathrm{NH}$ spectra obtained with the ASTRON and IUE satellites, the ammonia abundance was found to lie between 0.4 and $0.9 \%$, depending on the comet, suggesting a rather uniform $\mathrm{NH}_{3}$ relative abundance in comets (Feldman et al. 1993). Wyckoff et al. (1991a) reached a similar conclusion from the analysis of $\mathrm{NH}_{2}$ spectra in a sample of comets, although their inferred abundances were systematically lower (0.1-0.3 \%). Meier et al. (1994) recently reanalysed the ion mass spectrometer data of $\mathrm{P} /$ Halley and estimated the ammonia abundance to $1.5_{-0.7}^{+0.5} \%$, consistent with the range 0.4-0.9 \% derived by Feldman et al. 

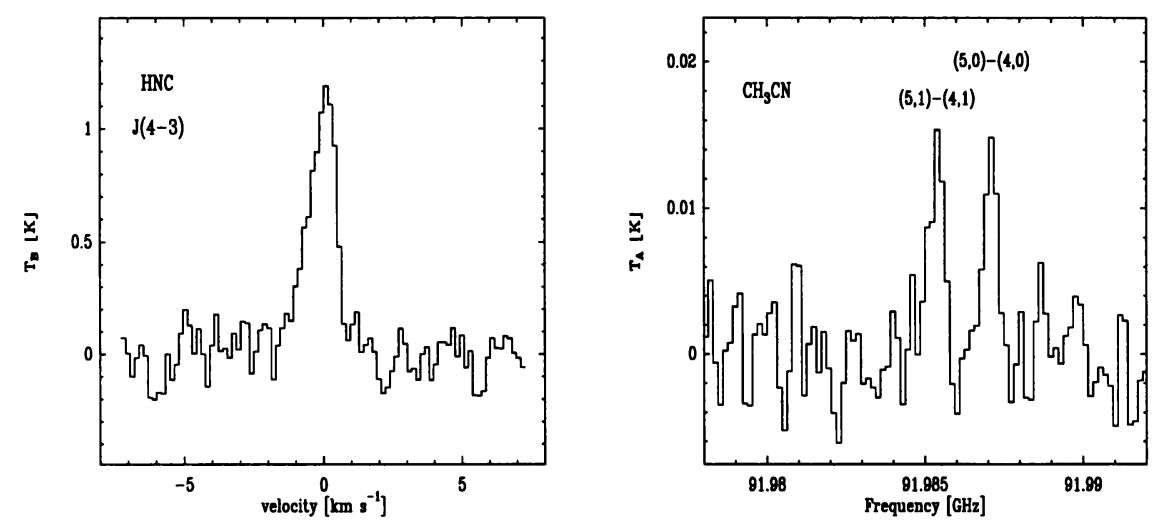

Figure 5. Left: The HNC J=4-3 line at $362.6 \mathrm{GHz}$ detected in comet C/1996 B2 Hyakutake on March 22, 1996 at the CSO (Irvine et al. 1996); Right: Detection of $\mathrm{CH}_{3} \mathrm{CN}$ at the IRAM Plateau de Bure Interferometer on March 26 (Dutrey et al. 1996).

(1993) for this comet, but significantly larger than the value found in comet Hyakutake.

HCN has been detected in several comets at millimetre and submillimetre wavelengths. Several rotational lines $(\mathrm{J}=1-0,3-2,4-3,8-7)$ have now been observed (e.g. Bockelée-Morvan 1994b; Despois et al. 1996b). In particular, the $\mathrm{J}=8-7$ line at $709 \mathrm{GHz}$ has been detected for the first time in comet Hyakutake using the Caltech Submillimeter Observatory (Lis et al. 1996b). Two individual lines of the $\nu_{3}$ band of HCN at $3 \mu \mathrm{m}$ were also detected in this comet (Fig. 3; Brooke et al. 1996). From the radio determinations, $\mathrm{HCN}$ is a minor compound of cometary atmospheres with abundances from 0.1 to $0.2 \%$. HCN is the main progenitor of the CN radicals seen at optical wavelengths, although we have several evidences that $\mathrm{CN}$ is partly produced from dust (A'Hearn et al. 1986).

The identification of HNC was made in comet Hyakutake from its $\mathrm{J}=4-3$ transition at $363 \mathrm{GHz}$ (Fig. 5). The detection obtained at JCMT (Matthews et al. 1996) was then securely confirmed at the CSO (Lis et al. 1996b). Both observations agree with a $\mathrm{HNC} / \mathrm{HCN}$ abundance ratio of about $6 \%$ (Irvine et al. 1996). The HNC abundance relative to water is about $0.01 \%$.

Finally, another nitrogen species firmly identified in comet Hyakutake is $\mathrm{CH}_{3} \mathrm{CN}$, from observations of several of its $(5, \mathrm{~K})-(4, \mathrm{~K})$ rotational lines at $92 \mathrm{GHz}$ using the IRAM Plateau de Bure interferometer in a single-dish mode (Fig. 5; Dutrey et al. 1996). The marginal detection of this species was claimed in comet C/Kohoutek (1973 XII) (Ulich \& Conklin 1974), but several other attempts were unsuccessful. The abundance inferred in 
comet Hyakutake is $0.01 \%$, in agreement with the upper limits obtained previously. $\mathrm{CH}_{3} \mathrm{CN}$ was detected in comet Hale-Bopp in August 1996, as the comet was at 3.3 AU from the Sun, using the IRAM 30-m telescope (Biver et al. 1996b).

$\mathrm{N}_{2}$ itself has not been detectable spectroscopically in comets, having no transitions accessible with available instrumentation. Mass spectrometer data yielded an upper limit on its abundance of $10-15 \%$ relative to water. Its abundance was estimated at $0.02 \%$ in comet $\mathrm{P} /$ Halley from observations of $\mathrm{N}_{2}^{+}$in the visible (Wyckoff et al. 1991b).

\subsubsection{Sulphur species}

$\mathrm{H}_{2} \mathrm{~S}$, OCS and $\mathrm{S}_{2}$ are the three sulphurous parent molecules identified by their spectral signatures in cometary comae.

$\mathrm{H}_{2} \mathrm{~S}$ was detected at millimetre wavelengths in several comets with abundances of the order of 0.2-0.3\% (e.g. Crovisier et al. 1991). Its abundance in comet $\mathrm{P} /$ Halley was investigated from ion mass spectrometer data (Geiss et al. 1991).

OCS, which showed a marginal signature in the IR spectrum of $\mathrm{P} / \mathrm{Halley}$ taken with the IKS/VEGA experiment (Combes et al. 1988), was definitely identified in comet Hyakutake at millimetre wavelengths (Woodney et al. 1996) with an abundance of $0.3 \%$ with respect to water.

$\mathrm{S}_{2}$ was first identified in 1983 during IUE observations of the Earthgrazing comet C/1983H1 IRAS-Araki-Alcock (A'Hearn et al. 1983). HST observations of comet Hyakutake provided the only detection since this initial discovery (Weaver et al. 1996a). The $\mathrm{S}_{2}$ abundance in cometary nuclei seems to be highly variable. The $\mathrm{S}_{2}$ abundance inferred in comet Hyakutake is of the order of $0.005 \%$, which is 4 times lower than the "quiescent" value measured in IRAS-Araki-Alcock, and 40 times lower than that measured during an outburst (Weaver et al. 1996b).

Finally, it seems likely that the CS radical, currently observed in the UV with IUE or the HST, comes from the $\mathrm{CS}_{2}$ molecule, with abundances on the order of $0.1 \%$ (Feldman et al. 1991). Indeed, its spatial distribution suggests photodissociation from a short-lived species and there are not many alternatives other than $\mathrm{CS}_{2}$. The detection of CS at millimetre and submillimetre wavelengths in comet Hyakutake (e.g. Matthews et al. 1996) opened the door for a detailed investigation from ground-based telescopes.

\subsection{COMET HALE-BOPP AT LARGE HELIOCENTRIC DISTANCES}

The discovery of the exceptionally bright comet Hale-Bopp in July 1995 at $7 \mathrm{AU}$ from the Sun, well before its perihelion (April 1, 1997), gave the first opportunity to measure molecular abundances in the coma at large 
heliocentric distances and to follow their evolution as the distance decreases. These data should allow to study the role of fractionation processes in the sublimation of cometary ices and can provide clues on the physical state of cometary ices. One of the major concerns is how the molecular abundances measured near $1 \mathrm{AU}$ from the Sun are related to the initial composition in the nucleus.

Table 1 shows the relative abundances measured in June 1996 at 4 AU from the Sun. These abundances present strong differences with those measured in comets near $1 \mathrm{AU} . \mathrm{CO}, \mathrm{CO}_{2}, \mathrm{H}_{2} \mathrm{~S}$, and $\mathrm{HCN}$ are overabundant, while $\mathrm{H}_{2} \mathrm{CO}$ is underabundant. In contrast, the abundances of $\mathrm{CH}_{3} \mathrm{OH}$ and $\mathrm{CS}$ are similar. Table 1 shows the sublimation temperature of these species. In general, the most volatile species are those which are overabundant in comet Hale-Bopp. But there is no direct correlation between volatility and overabundance. This probably reflects the complex phenomena taking place inside a comet nucleus upon solar heating. Due to the very porous nature of the nucleus, sublimation of volatiles may be triggered far below the surface and gas may diffuse inward and recondense at new places, causing chemical differentiation. Models treating the heat and gas diffusion inside a porous nucleus made of amorphous ice mixed with solid $\mathrm{CO}$ or $\mathrm{CO}_{2}$ have shown that molecular abundances in the coma at a given time strongly depend on the thermal history of the nucleus (Espinasse et al. 1991). It is also shown that the abundances of volatile species such as $\mathrm{CO}$ and $\mathrm{CO}_{2}$ measured at perihelion are significantly lower than the initial abundances in the nucleus.

\section{Outstanding issues}

We have reviewed the composition of cometary volatiles. The presence of volatile species such as $\mathrm{CO}, \mathrm{H}_{2} \mathrm{~S}$ or $\mathrm{N}_{2}$, the probable amorphicity of water ice and the possible low spin temperature of water are growing evidences that cometary ices condensed and remained in low temperature environments.

The chemical abundances measured in cometary comas can be examined in the context of either a solar nebula or an interstellar origin for the cometary material. In this study, we must re-emphasize the fact that these abundances probably do not reflect exactly the molecular abundances which were originally in the nucleus. This is particularly true for the most volatile species like $\mathrm{CO}$ or $\mathrm{H}_{2} \mathrm{~S}$.

The formation and condensation sequence of volatiles in an initially hot nebula with solar elemental abundances has been studied by Lewis \& Prinn (1980) and Fegley \& Prinn (1989). They showed that, due to kinetic inhibition of hydration processes, one would expect low-temperature condensates to be dominated by $\mathrm{CO}, \mathrm{CO}_{2}$ and $\mathrm{N}_{2}$ and contain insignificant 
amounts of $\mathrm{CH}_{4}, \mathrm{NH}_{3}$ and $\mathrm{HCN}$ and other carbon- and nitrogen- bearing species.

However, the $\mathrm{CH}_{4}$ to $\mathrm{CO}$ mixing ratio, the $\mathrm{HCN}$ and $\mathrm{NH}_{3}$ abundances and the presence of $\mathrm{H}_{2} \mathrm{CO}, \mathrm{CH}_{3} \mathrm{OH}$ and other organics in cometary material indicate that its origin may have to be considered in a different framework than condensation in a cooling solar nebula. In fact, the $\mathrm{D} / \mathrm{H}$ ratio in water of $310^{-4}$ measured in comets provides evidence that cometary ices formed from volatiles originating from interstellar grains mantles (Eberhardt et al. 1995; Balsiger et al. 1995; Gautier et al. 1996): this ratio, which is one order of magnitude larger than the protosolar value given by Geiss (1993), and twice the value of ocean water, is much higher than that which could have been reached through molecular isotopic exchanges in the solar nebula. It requires ion-molecule reactions which may only occur in the cold ISM. Strong deuterium enrichments similar to that measured in comets are observed in the hot cores of giant molecular clouds, which are thought to reflect evaporation of icy mantles of interstellar grains (e.g. Gensheimer et al. 1996).

What is the exact path between interstellar and cometary ices ? According to the scenario of Lunine et al. (1991), the ice-coated presolar grains falling into the solar nebula would have been heated by gas friction to such an extent that part of the icy mantles evaporated. The vapour would have then recondensed in an amorphous form onto the cold grains that have reached equilibrium with the nebular gas. Bar-Nun \& Kleinfeld (1989) have shown experimentally that, in this scenario, amorphous ices can trap large amounts of gases if condensed at very low temperatures. In particular $\mathrm{CO}: \mathrm{H}_{2} \mathrm{O}$ mixing ratios of a few percents are obtainable in the ices at a temperature of $50 \mathrm{~K}$ from a $\mathrm{CO} / \mathrm{H}_{2} \mathrm{O}$ gaseous mixture representative of evaporated mantles. According to Notesco \& Bar-Nun (1996), this scenario is also able to explain the strong depletion of $\mathrm{N}_{2}$ with respect to $\mathrm{CO}$ observed in comets.

A different scenario has been proposed by Yamamoto et al. (1983) and Yamamoto (1985). According to them, cometary ices are a sublimation residue of interstellar ices. From the comparison of the abundances of cometary and interstellar molecules, they showed that only the very volatile species $\left(\mathrm{N}_{2}\right.$ and $\left.\mathrm{CO}\right)$ were lost by sublimation. Greenberg (1982) and Greenberg \& Hage (1990) argued also, taking into account both ice and dust properties, that comets could be made of almost unprocessed interstellar material.

The composition of interstellar ices is now better known. New solid state features were recently detected in molecular clouds and protostellar objects using ISO (Boogert et al. 1996; d'Hendecourt et al. 1996; Whittet et al. 1996). They give growing evidences that cometary material has 
strong similarities with interstellar grains. Reported $\mathrm{CH}_{3} \mathrm{OH}, \mathrm{CO}$ and $\mathrm{CO}_{2}$ abundances are indeed comparable to or within a factor of 2-3 from those measured in comets. This is also true for the $\mathrm{CH}_{4}$ abundance which ranges from 0.4 to $4 \%$ in interstellar ices to be compared to the value of $0.7 \%$ measured in comet Hyakutake. Solid state features which could be due to $\mathrm{H}_{2} \mathrm{CO}$ and OCS were also observed (d'Hendecourt et al. 1996).

Interesting is the analogy with bipolar flows. In these regions, molecules are thought to be released from grain mantles. Despois et al. (1996c) showed that the $\mathrm{HCN}, \mathrm{HNC}, \mathrm{NH}_{3}, \mathrm{H}_{2} \mathrm{~S}$, OCS, $\mathrm{H}_{2} \mathrm{CO}$ and $\mathrm{CH}_{3} \mathrm{OH}$ relative abundances are in good agreement with those measured in comets. The only major discrepancy concerns the S-bearing species $\mathrm{SO}$ and $\mathrm{SO}_{2}$, which have very low abundance in comets (Table 1). Despois (1992a) and Despois et al. (1992b, 1996c) suggest that $\mathrm{H}_{2} \mathrm{~S}$ could be especially abundant in the ISM grains, as the result of grain-surface chemistry, and that hot gas chemistry would be responsible for the enhancement of $\mathrm{SO}$ and $\mathrm{SO}_{2}$ in bipolar flows.

Solid $\mathrm{C}_{2} \mathrm{H}_{2}$ has not been yet observed in the interstellar medium. As discussed by Brooke et al. (1996), a model for solid phase abundances in molecular clouds gives $\mathrm{C}_{2} \mathrm{H}_{2}$ abundances in close agreement with the cometary abundance. The high abundance of ethane observed in comet Hyakutake is puzzling. In contrast to $\mathrm{C}_{2} \mathrm{H}_{2}$, production of ethane by gasphase ion-molecule reactions is not expected in cold molecular clouds. $\mathrm{C}_{2} \mathrm{H}_{6}$ has never been observed in the ISM. Mumma et al. (1996a) proposes that ethane formed in the solid phase by hydrogenation of $\mathrm{C}_{2} \mathrm{H}_{2}$ or by photoprocessing of methane-rich ices. The abundance of $\mathrm{HNC}$ relative to $\mathrm{HCN}$ found in comet Hyakutake is strikingly reminiscent of the ratio observed in quiescent interstellar molecular clouds. Irvine et al. (1996) argues that this ratio might give additional evidence for comets preserving interstellar material. Finally, the origin of the very unstable molecule $S_{2}$ in comets is unclear. $S_{2}$ may have been produced in the presolar grains by UV irradiation (Grim \& Greenberg 1987) but this process produces also significant $\mathrm{SO}_{2}$ (Moore et al. 1988).

Whether cometary ices are the primordial icy mantles of the grains of the interstellar cloud or a second generation of ices recondensed in the solar nebula is still an open question. The strong analogy between cometary and ISM ices indicates at least that little chemical processing occurred between the collapse of the presolar cloud and the coagulation of the grains which formed the comets.

\section{References}

A'Hearn, M.F., Feldman, P.D., Schleicher D.G. 1983, ApJ 274, L29

A'Hearn, M.F., Hoban, S., Birch. P.V. et al. 1986, Nature 324, 649

Allen, M., Delitsky, M., Huntress, W. et al. 1987, A\&A 187, 502 
Altenhoff, W.J., Batrla, W., Huchtmeier W.K. et al. 1984, A\&A Letter, 125, L19

Balsiger, H., Altwegg, K., Geiss, J. 1995, J. Geophys. Res. 100, 5827

Bar-Nun, A., Kleinfeld, K. 1989, Icarus 80, 243

Biver, N., Bockelée-Morvan, D., Colom et al. 1996a, IAU Circ. 6421

Biver, N., Enzian, A., Bockelée-Morvan, D. et al. 1996b, IAU Circ. 6458

Bockelée-Morvan, D., Brooke, T.Y., Crovisier, J. 1995, Icarus 116, 18

Bockelée-Morvan, D., Crovisier, J. 1989 in Asteroids, Comets, Meteors III, ed. C.-I. Lagerkvist et al., Uppsala University, p. 267

Bockelée-Morvan, D., Crovisier, J., Colom, P., Despois, D. 1994a, A\&A 287, 647

Bockelée-Morvan, D., Crovisier, J., Despois, D. et al. 1987, A\&A 180, 253

Bockelée-Morvan, D., Padman, R., Davies, D.K., Crovisier, J. 1994b, Planet. Space Sci. 42,655

Boogert, A.C.A., Schutte, W.A., Tielens, A.G.G.M. et al. 1996, A\&A 315, L377

Brooke, T.Y., Tokunaga, A.T., Weaver, H.A. et al. 1996, Nature 383, 606

Cabot, H., Enzian, A., Klinger, J., Majolet, S. 1996, Planet. Space Sci 44, 1015

Combes, M., Moroz, V.I., Crovisier, J. et al. 1988, Icarus 76, 404

Crovisier, J. 1994, in Asteroids, Comets, Meteors 1993, IAU Symposium 160, ed. A. Milani et al. (Kluwer, Dordrecht), p. 313

Crovisier, J., Biver, N., Bockelée-Morvan, D. et al. 1995 Icarus 115, 213

Crovisier, J., Brooke, T.Y., Hanner, M.S. et al. 1996, A\&A 315, L385

Crovisier, J., Despois, D., Bockelée-Morvan, D. et al. 1991 Icarus 93, 246

Davies, J., Geballe, T., Cruishank, D., Owen, T., De Bergh, C. 1995, IAU Circ 6225

Despois, D. 1992a in Astrochemistry of cosmic phenomena, IAU Symposium 150, ed P.D. Singh (Kluwer, Dordrecht) p. 451

Despois, D., Bachiller, R., Crovisier, J. et al. 1996c Symposium UAI 178, in Asteroids, Comets, Meteors 1996, Versailles 8-12 July 1996, COSPAR Colloquium 10

Despois, D., Biver, N., Bockelée-Morvan, D. et al. 1996b, Planet. Space Sci 44, 529

Despois, D., Crovisier, J., Bockelée-Morvan, D., Colom, P. 1992b in Astrochemistry of cosmic phenomena, IAU Symposium 150, ed P.D. Singh (Kluwer, Dordrecht) p. 459

Despois, D., Wink, J., Neri, R., Lucas, R. et al. 1996a, IAU Circ 6388

d'Hendecourt, L., Jourdain de Muizon, M., Dartois, E. et al. 1996, A\&A 315, L365

DiSanti, M.A., Mumma, M.J., Geballe, T.R., Davies, J.K. 1995 Icarus 116, 1

Duncan, M., Quinn, T., Tremaine, S. 1988, ApJ 328, L69

Dutrey, A., Despois, D., Bockelée-Morvan, D. et al. IAU Circ 6364

Eberhardt, P., Krankowsky, D., Schulte, U. et al. 1987, A\&A 187, 481

Eberhardt, P., Meier, R., Krankowsky, D., Hodges, R.R. 1991, BAAS 23, 1161

Eberhardt, P., Reber, M., Krankowsky, D., Hodges, R.R. 1995, A\&A 302, 301

Edgeworth, K.E. 1949, MNRAS 109, 600

Enzian, A., Cabot, H., Klinger, J. 1996, A\&A, in press

Espinasse, S., Klinger, J., Ritz, C., Schmitt, B. 1991, Icarus 92, 350

Fegley, B. Jr., Prinn, R.G. 1989, in The Formation and Evolution of Planetary Systems, ed. H.A. Weaver, L. Danly, Cambridge University Press (Cambridge, England), p. 171

Feldman, P.D. 1991, in Comets in the Post-Halley Era, ed. R.L. Newburn et al. (Kluwer, Dordrecht), p. 139

Feldman, P. D., Festou, Rodriguez, P.M., Gonzalez, R. 1996, IAU Circ. 6370

Feldman, P.D., Fournier, K.B., Grinin, V. P., Zverera, A.M. 1993, ApJ 404, 348

Fernandez, J.A. 1980, MNRAS 192, 481

Festou, M.C., Rickman, H., West, R.M. 1993, A\&A Rev 4, 363, 5, 37

Gautier, D., Bockelée-Morvan, D., Lis D.C. et al. 1996, in Asteroids, Comets, Meteors 1996, Versailles 8-12 July 1996, COSPAR Colloquium 10

Geiss, J. 1993, in Origin and Evolution of the Elements, ed. N. Prantzos et al., Cambridge University Press (Cambridge, England), p. 89

Geiss, J., Altwegg, K., Anders, E. et al. 1991, A\&A 247, 226

Gensheimer, P.D., Mauersberger, R., Wilson, T.L. 1996, A\&A 314, 281 
Greenberg, J.M. 1982 in Comets, ed L.L. Wilkening, Univ. of Arizona Press, (Tucson), p. 131

Greenberg, J.M., Hage, J.I. 1990, ApJ 361, 260

Grim, R.J.A., Greenberg, J.M. 1987, ApJ 181, 155

Haider, S.A., Bhardwaj, A., Singhal, R.P. 1993, Icarus 101, 234

Hoban, S., Mumma, M.J., Reuter, D.C., DiSanti, M., Joyce, R.R. 1991, Icarus 93, 122

Huebner, W.F. 1987, Science 237, 628

Irvine, W., Bockelée-Morvan, D., Lis, D. et al 1996, Nature 382, 418

Jewitt, D.C., Luu, J.X. 1993, Nature 362, 730

Jewitt, D.C., Luu, J.X.,, Chen, J. 1996, AJ 112, 1225

Joblin, C., Boissel, P., de Parseval, P. 1996, Planet. Space Sci. (submitted)

Klinger, J., Ocampo, J., Schmitt, B. 1985 in Asteroids, Comets, Meteors II, ed. Lagerkvist et al. (Uppsala University, Uppsala), p. 241

Krankowsky, V.A., Lammerzahl, P., Herrwerth, I. et al. 1986, Nature 321, 326

Kuiper, G.P. 1951, in Astrophysics, ed. J.A. Hynek, p. 357

Lewis, J.S., Prinn, R.G. 1980, ApJ 238, 357

Lis, D.C., Keene, J., Young, K. et al. 1996a, IAU Circ 6362

Lis, D.C., Keene, J., Young, K. et al. 1996b, in Asteroids, Comets, Meteors 1996, Versailles 8-12 July 1996, COSPAR Colloquium 10

Lunine, J.I., Engel, S., Rizk, B., Horanyi, M. 1991, Icarus 94, 333

Matthews, H.E., Biver, N., Senay, M. et al. 1996 IAU Circ 6353

Meier, R., Eberhardt, P., Krankowsky, D., Hodges, R.R. 1993, A\&A 277, 677

Meier, R., Eberhardt, P., Krankowsky, D., Hodges, R.R. 1994, A\&A 287, 268

Moore, M.H., Donn, B.,, Hudson, R.L. 1988, Icarus 74, 399

Moreels, G., Clairemidi, J., Hermine, P., Brechignac P., Rousselot, P. 1994, A\&A 282,643

Mumma, M.J., Disanti, M.A., Tokunaga, A., Roettger, E.E. 1995, BAAS 27, 1144

Mumma, M.J., Disanti, M.A., Russo et al. 1996a, Science 272, 1310

Mumma, M.J., Disanti, M.A., Russo et al. 1996b, IAU Circ. 6366

Mumma, M.J., Weaver, H.A., Larson, H.P., Davis, D.S., Williams, M. 1986, Science 232, 1523

Mumma, M.J, Hoban, S., Reuter, D.C., DiSanti, M.A. 1993b, in Asteroids, Comets, Meteors 1993, IAU Symposium 160, LPI contribution 810, p.227

Mumma, M.J., Weaver, H.A., Larson, H.P. 1987, A\&A 187, 419

Mumma, M.J., Weissman, P.R., Stern, A.A. 1993a in Protostars and Planets III, ed E.H. Levy, J.I. Lunine (Univ. of Arizona Press, Tucson), p. 1177

Notesco, G., Bar-Nun, A. 1996, Icarus 122, 118

Schmitt, B., Espinasse, S., Klinger, J. 1993, in Workshop on the activity of distant comets, ed. W.F. Huebner et al. (Southwest Institute), p. 124

Senay, M.C., Jewitt D. 1994 Nature 371, 229

Ulich, B.L., Conklin, E.K. 1974, Nature 248, 121

Weaver, H.A., Feldman, P.D., McPhate, J.B. et al. 1994, ApJ 422, 374

Weaver, H.A., Feldman, P.D., A'Hearn, M.F. et al. 1996a, IAU Circ 6374

Weaver, H.A., Feldman, P.D., A'Hearn, M.F. et al. 1996b, in Asteroids, Comets, Meteors 1996, Versailles 8-12 July 1996, COSPAR Colloquium 10

Whittet, D.C.B., Schutte, W.A., Tielens, A.G.G.M. et al. 1996, A\&A 315, L357

Woodney, L.M., McMullin, J., A'Hearn, M.F. 1996, IAU Circ. 6344

Wootten, A., Butler, B., Bockelée-Morvan et al. 1996a, IAU Circ. 6362

Wootten, A., Butler, B., Bockelée-Morvan et al. 1996b in Asteroids, Comets, Meteors 1996, Versailles 8-12 July 1996, COSPAR Colloquium 10

Wyckoff, S.W., Tegler, S.C., Engel L. 1991a, ApJ 368, 279

Wyckoff, S.W., Tegler, S.C., Engel L. 1991b, ApJ 367, 641

Yamamoto, T. 1985, A\&A 142, 31

Yamamoto, T., Nakagawa, N., Fukui, Y. 1983, A\&A 122, 171 


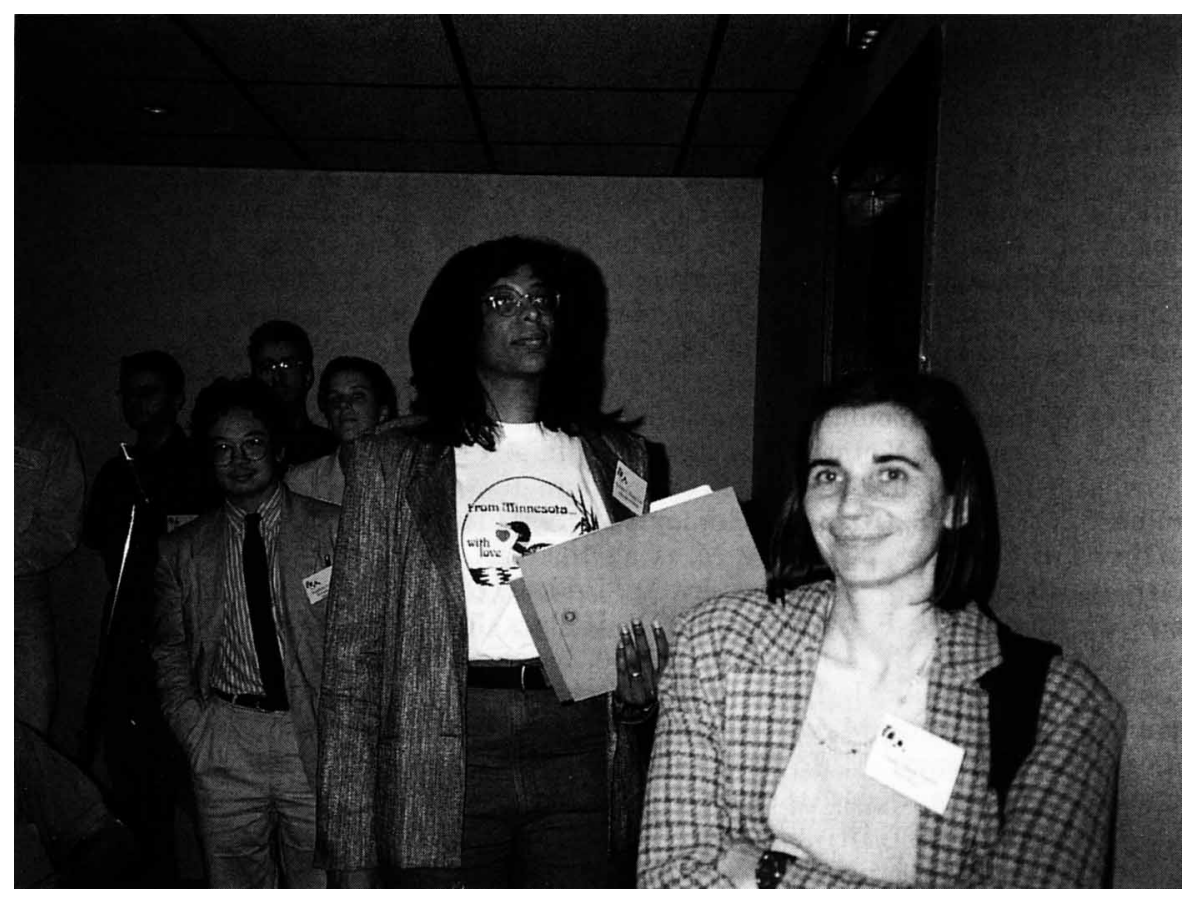

